\title{
Vasculitis allergica bei lymphoplasmozytischem Immunozytom
}

\begin{abstract}
Zusammenfassung. Bei einer 50-jährigen Patientin konnte erstmals zwei Jahre nach Manifestation einer chronisch-persistierend verlaufenden Vasculitis allergica ein lymphoplasmozytisches Immunozytom diagnostiziert werden. Die onkologische Therapie erfolgte daraufhin initial mit einer Zytostase nach dem CHOP-Schema. Bereits nach dem zweiten Zyklus konnte eine komplette Remission des Lymphoms festgestellt werden, während die Vasculitis allergica nur in ihrer klinischen Ausprägung rückläufig war. Nach dem sechsten Zyklus der Zytostase wurde die onkologische Therapie mit alpha-Interferon fortgesetzt. Zu diesem Zeitpunkt wurde die Patientin nun auch von Seiten ihrer Dermatose erscheinungsfrei. Bei einer Nachbeobachtungszeit von bisher 12 Monaten ist es zu keinem neuen Rezidiv der Vasculitis allergica gekommen. Die Kombination eines malignen Tumors mit einer Vasculitis allergica ist ein insgesamt seltenes Geschehen, wobei die Plasmozytome zahlenmäßig noch am häufigsten beobachtet wurden. Auf die in der Literatur gefundenen mit einer Vasculitis allergica assoziierten Malignome wird hingewiesen.
\end{abstract}

Allergic Vasculitis in a Patient with Lymphoplasmacytic Immunocytoma. Two years after the initial manifestation of a chronically persisting allergic vasculitis a lymphoplasmacytic immunocytoma was diagnosed in a 50 -year-old female patient. Cancer chemotherapy was initiated according to the CHOP-regimen. Complete remission of the lymphoma was already achieved after the second chemotherapeutic cycle, whereas the allergic vasculitis was only gradually declining. After the sixth cycle the therapy was continued with interferon alpha. At that time the dermatosis was also completely healed. Now, twelve months later, there hasn't been any relapse of the vasculitis. The coincidence of a malignant tumor and a leukocytoclastic vasculitis is a rare event with a malignant myeloma being the most frequently detected neoplasm. Other in the literature reported malignant tumors associated with an allergic vasculitis are mentioned.

Akt Dermatol 2001; 27: $93-96$

(c) Georg Thieme Verlag Stuttgart · New York

ISSN 0340-2541

\author{
G. Wagner \\ Hautklinik Zentralkrankenhaus Bremerhaven
}

\section{Einleitung}

Die Vasculitis allergica ist pathogenetisch das typische Beispiel einer primär-kutan auftretenden Immunkomplex-Vaskulitis. Histopathologisch finden sich dabei fibrinoide Gefäßwandnekrosen, eine meist ausgeprägte Leukozytoklasie und Erythrozytenextravasate, während in der direkten Immunfluoreszenz perivaskulär bei frischen Läsionen Immunglobuline und Komplementfaktoren nachweisbar sind [6,20,24]. Das klinische Bild der Vasculitis allergica ist gekennzeichnet durch eine palpable Purpura, durch Ulzera oder durch persistierende Urticae. Unabhängig vom klinischen Erscheinungsbild sind bei der Auslösung zahlreiche ätiologische Faktoren $\mathrm{zu}$ berücksichtigen. Bei Kindern und jungen Erwachsenen finden sich gehäuft bakterielle oder virale Infektionen, z.B. mit Streptokokken, Staphylokokken und Mycobakterien sowie mit Influenza-Viren, Mycoplasmen oder den Erregern der Hepatitis B und C $[2,16,21,30]$. Neben dieser infektallergischen Genese werden auch zahlreiche Arzneimittel für die Provokation einer Vasculitis allergica verantwortlich gemacht. Dabei gehören Antibiotika und nicht-steroidale Antiphlogistika zu den häufiger beschuldigten Medikamenten, während nur selten Digitalispräparate, Retinoide oder Neuroleptika als auslösende Substanzgruppen beschrieben worden sind $[17,28,29]$. Auch Lebensmittel, Lebensmitteladditiva und Gewürze können gelegentlich als Ursache einer Vasculitis allergica infrage kommen [5]. Weitere ätiologische Verknüpfungen bestehen zu den rheumatischen Erkrankungen und zu den Kollagenosen [1]. Nur ganz vereinzelt wurde eine Vasculitis allergica im Zusammenhang mit einer malignen Tumorerkrankung beobachtet. Dass insbesondere der chronische Verlauf einer Vasculitis allergica als Hinweis auf eine zugrunde liegende Neoplasie gewertet werden kann, soll am Beispiel der nachfolgenden Kasuistik gezeigt werden.

\section{Kasuistik}

Pat.: E. K., 50 Jahre, Hausfrau

\section{Anamnese}

Seit dem Dezember 1996 bemerkte die Patientin wenig juckende Hautveränderungen an den unteren Extremitäten. Die einzelnen Morphen bestanden jeweils für einige Wochen, um anschließend unter Hinterlassung diskreter Pigmentierungen wieder abzuheilen. Im Juni 1997 wurde die Patientin in der 
Hautklinik Bremerhaven vorgestellt. Zu diesem Zeitpunkt fanden sich an den unteren Extremitäten und gluteal beidseits disseminiert stehende, linsen- bis münzgroße, diskret infiltrierte, unscharf begrenzte Erytheme, die unter dem Glasspatel eine purpuriforme Pigmentierung aufwiesen. Daneben zeigten sich gleich große, etwas unterschiedlich braun pigmentierte Maculae (Abb. 1 u. 2).

\section{Histologie}

In der histopathologischen Untersuchung fanden sich die typischen Zeichen einer ausgeprägten leukozytoklastischen Vaskulitis, während in der direkten Immunfluoreszenz IgG- und C 3-Ablagerungen nachgewiesen werden konnten. Die Ätiologie der Vasculitis allergica blieb zunächst unklar. Die Arzneimittelanamnese war unauffällig. Hinweise für chronische Infekte ergaben sich ebenfalls nicht.

\section{Laborbefunde}

Mit Ausnahme einer mittelgradig erhöhten BSG von 48/56 mm waren alle routinemäßig bestimmten Laborparameter unauffällig (BB und Diff-BB, Transaminasen, nierenpflichtige Substanzen, Elektrolyte, CRP, ASL-Titer, Rheumafaktoren, Eiweißelektrophorese und Urinsediment). Darüber hinaus ergaben sich auch in der erweiterten Labordiagnostik keine pathologischen Befunde (ANA, ANCA, Antikardiolipin-AK, Komplementfaktoren, Kryoglobuline und Immunelektrophorese). Hinweise für akute oder persistierende virale oder bakterielle Infektionen zeigten sich weder laborchemisch noch, soweit durchführbar, mikrobiologisch (Hepatitis B und C, Chlamydien, Mycoplasmen, Salmonellen, Shigellen, Yersinien sowie Streptokokken, Helicobacter pylori und Borrelien).

\section{Weitere Befunde}

Auch bei den übrigen technischen Untersuchungen konnten keine pathologischen Befunde erhoben werden (Rö.-Thorax, Oberbauchsonographie, CT-Thorax und Abdomen). Des Weiteren konnten bei den konsiliarischen Vorstellungen in der HNO, MKG und Gynäkologie entzündliche Fokalgeschehen ausgeschlossen werden.

\section{Therapie und Verlauf}

Bei somit zunächst ungeklärter Ätiologie erfolgte die Behandlung initial mit $50 \mathrm{mg}$ Prednisolon täglich, was zu einer schnellen Rückbildung der kutanen Symptomatik führte. Die in den nachfolgenden Monaten wiederholt durchgeführten Versuche einer Dosisreduktion waren jedoch regelmäßig von Rezidiven begleitet. Auch die zusätzliche Behandlung mit Azathioprin oder Dapson erlaubte keine Dosisreduktion der Glukokortikoidtherapie unter $30 \mathrm{mg}$ täglich. Im Dezember 1998, somit 2 Jahre nach der Erstmanifestation der Vasculitis allergica, wurden die laborchemischen, mikrobiologischen und technischen Untersuchungen komplett wiederholt. In der Eiweißelektrophorese zeigte sich jetzt erstmals eine deutliche Erniedrigung der Gamma-Globulin-Fraktion mit einem Wert von 7,5\% (NW $11-19 \%$ ). Gleichzeitig fand sich in der Immunelektrophorese die folgende Befundkonstellation: IgG 6,2 g/l (NW 8-18 g/l), IgA 0,6 g/l (NW 0,9-4,5 g/l) und IgM 4,07 g/1 (NW 0,6-2,8 g/l). Keine Pathopräzipitate erkennbar, kein Anhalt für Defekt- oder Paraproteinämie. In der Urinelektropho-

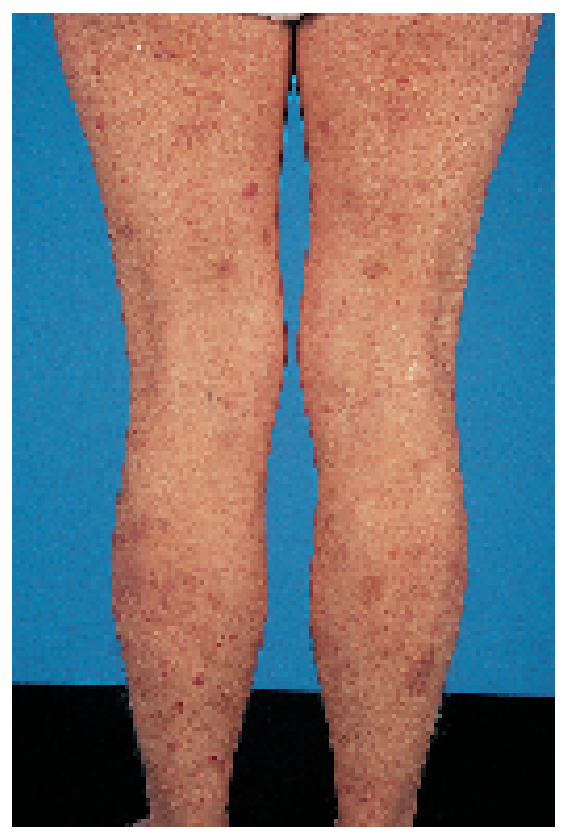

Abb. 1 Vasculitis allergica untere Extremitäten dorsal.

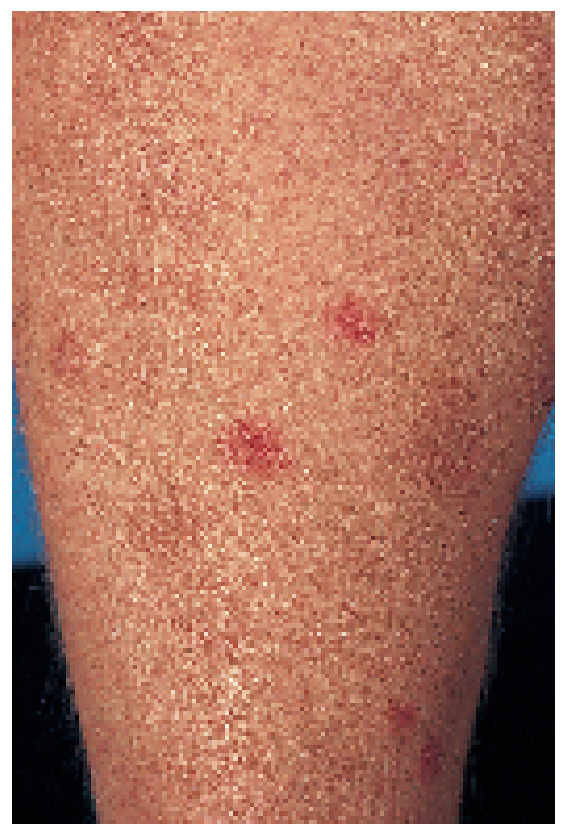

Abb. 2 Vasculitis allergica Oberschenkel Detailaufnahme.

rese kein Hinweis für Bence-Jones-Protein. In der nun durchgeführten Beckenkammbiopsie konnte histopathologisch der folgende Befund erhoben werden: Ausgedehnte lnfiltration des Knochenmarks durch ein niedrig malignes B-Zell-Lymphom, bei insgesamt knotigem Wachstum bestehend aus kleinen Lymphozyten und einzelnen Mastzellen. Immunhistochemisch Lymphozyten positiv für B-Zell-Antigen CD 20 sowie zytoplasmatisch monotypische Restriktion für leichte Kette lambda. Diagnose: Lymphoplasmozytisches Immunozytom. Internistischerseits erfolgte die onkologische Therapie initial nach dem CHOP-Schema. Bereits nach dem 2. von insgesamt 6 Zyklen zeigte sich bei einer Kontrollbiopsie des Knochenmarks eine komplette Remission des lymphoplasmozytischen Immunozytoms. Auch die Vasculitis allergica war während dieser Therapiephase klinisch deutlich rückläufig, eine voll- 
ständige Abheilung konnte jedoch noch nicht beobachtet werden. Erst nachdem die Therapie mit dem CHOP-Schema im Mai 1999 beendet wurde und die Patientin anschließend eine Behandlung mit 3 x 5 Mio. I.E. alpha-Interferon pro Woche erhielt, zeigten sich auch keine weiteren Exazerbationen der Vasculitis allergica. Bei einer Nachbeobachtungszeit von nunmehr 12 Monaten nach Einleitung der alpha-Interferon-Therapie ist die Patientin bisher von Seiten ihrer dermatologischen und onkologischen Symptomatik erscheinungsfrei geblieben.

\section{Diskussion}

Im Einzelfall können zahlreiche ätiologische Faktoren für das Auftreten einer Vasculitis allergica verantwortlich gemacht werden. Bakterielle oder virale Erkrankungen, Nahrungsmittelallergene, Medikamente sowie Erkrankungen aus dem rheumatischen Formenkreis einschließlich der Kollagenosen werden dabei am häufigsten als auslösende Ursachen angesehen $[2,12]$. Als Ausdruck einer kutanen Paraneoplasie wird die Vasculitis allergica hingegen nur selten beobachtet. Bei einer retrospektiven Analyse der Krankheitsdaten von 161 Patienten mit einer Vasculitis allergica fand Boslet bei 8 Patienten die zusätzliche Diagnose eines malignen Tumors, entsprechend einer Häufigkeit von 4\% [2]. Zum gleichen Ergebnis von ebenfalls $4 \%$ führten auch die Untersuchungen von Jessop, der bei 3 von 69 Patienten die Kombination einer Vasculitis allergica mit einem Malignom nachweisen konnte [12]. Eine mit etwa $6 \%$ etwas höhere Häufigkeit ermittelten Marques et al. bei einer Untersuchung von 51 Patienten, von denen drei eine tumorassoziierte Vasculitis allergica aufwiesen [18]. Ebenfalls hier zu erwähnen, jedoch im Gegensatz zu den bereits zitierten Arbeiten ohne die Möglichkeit einer prozentualen Häufigkeitsangabe, sind die Ergebnisse von Sanchez-Guerrero et al. Die Autoren fanden in einer Gruppe von 222 Vaskulitis-Patienten, deren jeweilige Vaskulitisformen in der Arbeit jedoch nicht speziell aufgeführt wurden, acht Patienten mit einer kutanen Vasculitis allergica bei einem gleichzeitig bestehenden malignen Tumor [23].

Bei der paraneoplastischen Vasculitis allergica sind in der Literatur unterschiedliche Malignome beschrieben worden, wobei die Non-Hodgkin-Lymphome am häufigsten beobachtet wurden. Innerhalb dieser Gruppe wiederum sind die Plasmozytome und die Haarzell-Leukämie auffällig zahlreich vertreten gewesen, während über andere Formen der Lymphome, aber auch über die Leukämien meist nur in einzelnen Kasuistiken berichtet worden ist. Letzteres trifft auch für die wenigen soliden Malignome zu, die in Verbindung mit einer Vasculitis allergica diagnostiziert werden konnten. Dabei fanden sich überwiegend Karzinome in gastrointestinaler oder urogenitaler Lokalisation. Eine Zusammenstellung der in der Literatur gefundenen, mit einer Vasculitis allergica assoziierten Malignome zeigt Tab.1. Auf welche Art und Weise ein maligner Tumor die Auslösung einer Vasculitis allergica initiiert, ist nicht geklärt. Dabei wird die Möglichkeit eines allergenwirksamen Tumorproteins diskutiert [4,13]. Bei einzelnen, jedoch nicht bei allen Patienten mit Lymphomen oder Leukämien konnten Kryoglobuline nachgewiesen werden, die als auslösende Ursache der Vasculitis allergica angesehen worden sind $[8,10,11,19]$. Bei den zahlreichen ätiologischen Möglichkeiten, die in jedem einzelnen Fall für das Auftreten einer Vasculitis allergica verantwortlich sein können, ergibt sich die Frage nach klinischen Anhaltspunkten, die möglicherweise auf eine
Tab.1 Vasculitis allergica assoziierte Malignome

Lymphome/Leukämien

Plasmozytom

$[10,11,14,15,19]$

Haarzell-Leukämie

$[9,22]$

diffuses großzelliges B-Zell-Lymphom

M. Hodgkin

akute/chronische myelotische Leukämie

Solide Malignome

Ösophaguskarzinom

Kolonkarzinom

Prostatakarzinom

Hodenteratom

Ovarialkarzinom

Mammakarzinom

Bronchialkarzinom

[3]

malignomassoziierte Auslösung hindeuten. Dabei scheint der chronische Verlauf einer Vasculitis allergica, besonders der chronisch-persistierende ein wichtiger Hinweis zu sein, der an einen malignen Tumor denken lassen sollte [8,23]. Dies gilt umso mehr, wenn alle anderen Ursachen einer Vasculitis allergica bereits ausgeschlossen werden konnten. Darüber hinaus kann auch die ungewöhnliche Therapieresistenz einer Vasculitis allergica auf ein zugrunde liegendes Malignom hinweisen. Des Weiteren sollte auf unspezifische klinische Symptome einer malignen Tumorerkrankung geachtet werden. Vor dem Hintergrund der gehäuft auftretenden Lymphome zählen hierzu Nachtschweiß, Gewichtsabnahme und Fieber sowie laborchemisch unklare BSG-Erhöhungen und pathologische Befunde in der Eiweißelektrophorese. Bei der Betreuung entsprechender Patienten ist ferner der Umstand zu berücksichtigen, dass die Vasculitis allergica auch über einen längeren Zeitraum bestehen kann, während das assoziierte Malignom trotz entsprechender Diagnostik noch nicht erfassbar ist $[8,10,23]$. Im Gegensatz zu den bisher erwähnten klinischen Hinweisen können die Ausdehnung, die Lokalisation und die jeweilige klinische Manifestationsform einer Vasculitis allergica nicht als spezifische Kriterien gewertet werden, die gegebenenfalls für einen malignen Tumor als auslösende Ursache sprechen würden $[4,7,10,27]$.

Die Kasuistik der hier vorgestellten Patientin ist in vielerlei Hinsicht als typisch anzusehen. Neben einem chronisch-persistierenden Verlauf und einer ungewöhnlichen Therapieresistenz konnte die Ursache der Vasculitis allergica zunächst nicht geklärt werden. Erst ein Jahr nach der primären Manifestation der Dermatose zeigte sich bei einer Wiederholung der technischen Untersuchungen eine Abweichung in der Eiweißelektrophorese, die dann letztendlich zur Diagnose eines lymphoplasmozytischen Immunozytoms führte, eines niedrig-malignen Non-Hodgkin-Lymphoms der B-Zell-Reihe. Der therapiebedingten kompletten Remission des Lymphoms folgte, wenn auch zeitlich verzögert, die Abheilung der Vasculitis allergica. Als Fazit bleibt abschließend festzustellen, dass das mögliche biologische Verhalten einer malignomassoziierten Vasculitis allergica regelmäßige klinische und technische Untersuchungen derjenigen Patienten rechtfertigt, bei denen die Ätiologie einer chronisch verlaufenden Vasculitis allergica nicht geklärt ist. 


\section{Literatur}

${ }^{1}$ v Bierbrauer A, Schmidt JA, v Wichert P. Die Bedeutung von Vaskulitiden bei Erkrankungen des rheumatischen Formenkreises. Internist 1993; 34: 632 - 647

2 Boslet WTh. Vasculitis allergica - eine retrospektive Analyse von 161 Patienten. Akt Dermatol 1995; 21: 381 - 385

${ }^{3}$ Cairns SA, Mallick NP, Lawler W, Williams G. Squamous cell carcinoma of bronchus presenting with Henoch-Schönlein purpura. Br Med J 1978; 2: 474 - 475

${ }^{4}$ Callen JP. Cutaneous leukocytoclastic vasculitis in a patient with an adenocarcinoma of the colon. J Rheumatol 1987; 14: 386 389

${ }^{5}$ Eisenmann A, Ring J, v d Helm D, Meurer M, Braun-Falco O. Vasculitis allergica durch Nahrungsmittelallergie. Hautarzt 1988; 39: 318 - 321

${ }^{6}$ Ekenstam EA, Callen JP. Cutaneous leukocytoclastic vasculitis. Arch Dermatol 1984; 120: 484 - 489

${ }^{7}$ Garcias VA, Herr HW. Henoch-Schönlein purpura associated with cancer of prostate. Urology 1982; 19: 155 - 158

${ }^{8}$ Greer JM, Longley S, Edwards NL, Elfenbein GJ, Panush RS. Vasculitis associated with malignancy. Medicine 1988; 67: 220 - 230

${ }^{9}$ Hasler P, Kistler H, Gerber H. Vasculitides in hairy cell leukemia. Semin Arthritis Rheum 1995; 25: 134 - 142

${ }^{10}$ Hennecke Th, Harlacher R, Kemper R, Neumann R, Kindler J. 50jähriger Patient mit leukozytoklastischer Vaskulitis und Arthralgien. Internist 1996; 37: 1159-1162

${ }^{11}$ Highet AS. Urticarial vasculitis and IgA myeloma. Br J Dermatol 1980; 102: 355 - 357

12 Jessop SJ. Cutaneous leukocytoclastic vasculitis: A clinical and aetiological study. Br J Rheumatol 1995; 34: 942 - 945

${ }^{13}$ Kesseler ME. Cutaneous vasculitis: A presenting feature of Hodgkin's disease. J R Soc Med 1986; 79: 485 - 486

${ }^{14}$ Kois JM, Mack Sexton F, Lookingbill DP. Cutaneous manifestations of multiple myeloma. Arch Dermatol 1991; 127: 69 - 74

${ }^{15}$ Landthaler M, Scherer R, Wolff HH. Kryoglobulinämie bei Plasmozytom. Hautarzt 1980; 31: 665 - 667

${ }^{16}$ Lee A, Jang J, Lee K. Two cases of leukocytoclastic vasculitis with tuberculosis. Clin Exp Dermatol 1998; 23: 225 - 226

${ }^{17}$ Lee AY. A case of leukocytoclastic vasculitis associated with haloperidol. Clin Exp Dermatol 1999; 34: 430 - 432

${ }^{18}$ Marques C, Sereijo M, Domingues JC, Sequeira J, Baptista AP. Vasculite leucocitoclasica. Revisao de 51 casos. Acta Med Port 1995; 8: $15-22$

${ }^{19}$ McMillen JJ, Krueger SK, Dyer GA. Leukocytoclastic vasculitis in association with immunglobulin A myeloma. Ann Intern Med 1986; 105: 709 - 710

${ }^{20}$ Mensing H. Leukozytoklastische Vaskulitis. Immun Infekt 1987; 15: 9 - 14

${ }^{21}$ Perez C, Mendoza H, Hernandez R, Valcayo A, Guarch R. Leukocytoclastic vasculitis and polyarthritis associated with Mycoplasma pneumoniae. Clin Infekt Dis 1997; 25: 154 - 155

22 Pope A, Lazarchick J, Hoyer L, Weinstein A. Hairy cell leukemia and vasculitis. J Rheum 1980; 7: 895 - 899

${ }^{23}$ Sanchez-Guerrero J, Gutierrez-Urena S, Vidaller A, Reyes E, Iglesias A, Alarcon-Segovia D. Vasculitis as a paraneoplastic syndrome. Report of 11 cases and review of the literature. J Rheumatol 1990; 17: 1458 - 1462

${ }^{24}$ Sneller MC, Fauci AS. Pathogenesis of vasculitis syndromes. Adv Rheumatol 1997; 81: 221 - 242

${ }^{25}$ Sproßmann A, Müller RPA. Urtikaria-Vaskulitis-Syndrom bei metastasierenden malignen Hodenteratom. Hautarzt 1994; 45: $871-874$

${ }^{26}$ Stashower ME, Rennie TA, Turiansky GW, Gilliland WR. Ovarian cancer presenting as leukocytoclastic vasculitis. J Am Acad Dermatol 1999; 40: 287 - 289
${ }^{27}$ Vesole DH. Diffuse large-cell lymphoma in a adult with Schönlein-Henoch purpura. Arch Intern Med 1987; 147: 2026 - 2027

${ }^{28}$ Wagner G, Lubach D. Über die Vasculitis allergica als unerwartete Nebenwirkung einer Digitalis-Therapie. Akt Dermatol 1986; 12: $125-127$

${ }^{29}$ Wagner G. Vasculitis allergica bei Retinoid-Therapie einer Acne conglobata. Akt Dermatol 2000; 26: 160 - 163

${ }^{30}$ Wagner G, Schmeck-Lindenau HJ. Vasculitis allergica bei Hepatitis-C-induzierter Kryoglobulinämie. Akt Dermatol, im Druck

\section{Dr. G. Wagner}

Chefarzt der Hautklinik

Zentralkrankenhaus Reinkenheide

Postbrookstraße 103

27574 Bremerhaven 